\title{
Evidence for distinct levels of neural adaptation to both coherent and incoherently moving visual surfaces in visual area hMT+
}

\author{
Teresa Sousa $^{\text {a,b,c,1 }}$, Alexandre Sayal ${ }^{\text {a,b,c,1 }}$, João V. Duarte ${ }^{\text {a,b,c }}$, Gabriel N. Costa ${ }^{\text {a,b,c }}$, \\ Ricardo Martins ${ }^{\text {a,b,c, Miguel Castelo-Branco }}{ }^{\text {a,b,c, d,** }}$ \\ ${ }^{a}$ Coimbra Institute for Biomedical Imaging and Translational Research (CIBIT), University of Coimbra, Portugal \\ ${ }^{\mathrm{b}}$ Institute of Nuclear Sciences Applied to Health (ICNAS), University of Coimbra, Portugal \\ ${ }^{\mathrm{c}}$ Institute for Biomedical Imaging and Life Sciences (CNC.IBILI), Faculty of Medicine, University of Coimbra, Portugal \\ ${ }^{\mathrm{d}}$ ICNAS - Produção, University of Coimbra, Portugal
}

\section{A R T I C L E I N F O}

\section{Keywords:}

Bistable visual motion

Perceptual decision

Adaptation

Plaids

hMT+

\begin{abstract}
A B S T R A C T
Visual adaptation describes the processes by which the visual system alters its operating properties in response to changes in the environment. It is one of the mechanisms controlling visual perceptual bistability - when two perceptual solutions are available - by controlling the duration of each percept. Moving plaids are an example of such ambiguity. They can be perceived as two surfaces sliding incoherently over each other or as a single coherent surface.

Here, we investigated, using fMRI, whether activity in the human motion complex (hMT+), a region tightly related to the perceptual integration of visual motion, is modulated by distinct forms of visual adaptation to coherent or incoherent perception of moving plaids. Our hypothesis is that exposure to global coherent or incoherent moving stimuli leads to different levels of measurable adaptation, reflected in hMT + activity. We found that the strength of the measured visual adaptation effect depended on whether subjects integrated (coherent percept) or segregated (incoherent percept) surface motion signals.

Visual motion adaptation was significant both for coherent motion and globally incoherent surface motion. Although not as strong as to the coherent percept, visual adaptation due to the incoherent percept also affects hMT + . This shows that adaptation can contribute to regulate percept duration during visual bistability, with distinct weights, depending on the type of percept. Our findings suggest a link between bistability and adaptation mechanisms, both due to coherent and incoherent motion percepts, but in an asymmetric manner. These asymmetric adaptation weights have strong implications in models of perceptual decision and may explain asymmetry of perceptual interpretation periods.
\end{abstract}

\section{Introduction}

After prolonged exposure to a stimulus, neuronal responses tend to adjust to a new level. This phenomenon, known as adaptation (Gibson, 1937; Kohn, 2007; Solomon and Kohn, 2014), has been described at the level of individual neurons (Kar and Krekelberg, 2016; Kohn and Movshon, 2003; Müller et al., 1999; Petersen et al., 1985; van Wezel and Britten, 2002) and of neuronal populations, as measured with functional magnetic resonance imaging (fMRI) (Castelo-Branco et al., 2009; Culham et al., 1999; Hogendoorn and Verstraten, 2013; Huk and Heeger, 2002; Larsson and Harrison, 2015; Tootell et al., 1995a). It underlies the observation that robustly activated neurons show reduced response when stimulated repeatedly, resulting in smaller amplitude of the measured neurophysiological signal (Grill-Spector et al., 2006; Krekelberg et al., 2006; Tootell et al., 1998).

In the motion domain, single cell recordings in the middle temporal (MT) visual cortex of monkeys have shown that, following adaptation to a moving pattern, there is an induced imbalance in the activation of directional-selective neurons (Kohn and Movshon, 2003; Petersen et al., 1985; van Wezel and Britten, 2002). This finding provides a plausible mechanism for the motion after-effect (MAE), a perceptual phenomenon in which a stationary stimulus, after a period of prolonged observation of

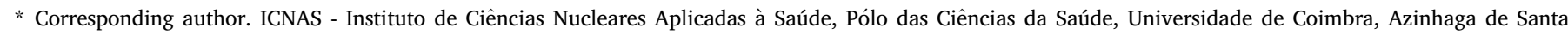
Comba, 3000-548, Coimbra, Portugal.

E-mail address: mcbranco@fmed.uc.pt (M. Castelo-Branco).

1 Both authors contributed equally.
} 
motion, is perceived moving in the opposite direction (Anstis et al., 1998; Mather et al., 2008). Therefore, when cells are exposed to a stimulus to which they respond strongly, the amount of adaptation is commonly associated with the subsequent strength of MAE (Anstis et al., 1998; Hershenson, 1989; Hogendoorn and Verstraten, 2013; Tootell et al., 1995a). Moreover, the existence of stimulus-selective adaptation effects in a brain region is interpreted as evidence for selective processing in that region (Grill-Spector et al., 2006; Grill-Spector and Malach, 2001; Mather et al., 2008).

The human motion complex (hMT+), homologous to the monkey MT region, is well known to be involved in motion perception (Kolster et al., 2010; Tootell et al., 1995b). Electrophysiological studies at the single-cell level in monkeys reported pattern and component motion selectivity in MT (Albright and Stoner, 1995; Movshon et al., 1985). Using plaids, i.e. bistable stimuli consisting of two moving gratings superimposed in the same display (Adelson and Movshon, 1982), we have previously shown in humans that hMT + underlies the perceptual integration of pattern (coherent) motion vs. segregation of component (incoherent) plaid motion signals (Castelo-Branco et al., 2002). Furthermore, Huk and Heeger used an fMRI adaptation paradigm to demonstrate that hMT + is selective for coherent plaid motion (Huk and Heeger, 2002). In fact, there is literature indicating neuronal adaptation as a possible cause of reduced perceived coherence and perceptual switches during bistable visual stimulation (Kohn and Movshon, 2004; Long and Toppino, 2004; Patterson et al., 2014). However, there is no neurophysiological evidence that adaptation to incoherent surface motion occurs.

Here, we aim to test, by using an fMRI adaptation paradigm, if $\mathrm{hMT}+$ adapts to motion signals arising from incoherent plaid motion and how they relate to coherent motion adaptation. We hypothesize that hMT + adapts differently to the coherent and incoherent motion percepts. This is relevant for the understanding of perceptual bistability - a perceptual phenomenon during which visual perception may alternate between competing interpretations of a physically unchanging stimulus (Leopold and Logothetis, 1999; Wang et al., 2013). If visual adaptation due to incoherent surface motion also occurs, it might be modulating the perceptual switches from incoherent to coherent motion as well. Accordingly, quantitative models of perceptual bistability (Huguet et al., 2014) depend on the relative importance of distinct adaptation mechanisms vs. noise when controlling perceptual choice and duration. Furthermore, different adaptation strengths/weights due to coherent and incoherent surface motion might explain the imbalance between percepts' duration during perceptual bistability.

We used a moving plaid, which can be seen as two surfaces - two gratings sliding over each other and moving in different directions (incoherent or component motion) - or as a single surface moving coherently - the two component surfaces are integrated perceptually into a single surface moving coherently in a direction intermediate to the directions of the individual component gratings (coherent or pattern motion). Taking advantage of the fact that perceptual motion coherency/ incoherency can be induced by adding background texture dots to the plaids, we studied the effect of adaptation to each motion percept in $\mathrm{hMT}+$, when compared to a non-adapting condition, while applying an attention control task.
We found that the measured visual adaptation effect depended on whether subjects integrated or segregated motion signals and was significant in both cases. These may shed light on the understanding of how the visual system achieves perceptual decisions based on low-level adaptation mechanisms.

\section{Methods}

\subsection{Participants}

Twenty healthy participants were recruited for this experiment (13 male, mean age $27.70 \pm 4.20$ ). All had normal or corrected-to-normal vision and no history of neurological or psychiatric diseases. The participants were right-handed, as confirmed by a handedness questionnaire adapted from (Oldfield, 1971). All gave informed written consent before participating, in accordance to the declaration of Helsinki, and the study complied with the safety guidelines for magnetic resonance imaging (MRI) research on humans. The work was approved by the Ethics Committee of the Faculty of Medicine of the University of Coimbra.

\subsection{Experimental setup}

We acquired in one session a structural MRI sequence and six fMRI sequences (a hMT+ functional localizer, four testing runs and a MAE control run - Fig. 1A) from all participants.

Stimuli were created in MATLAB R2016b (The Mathworks, Inc., Natick, MA-USA), along with the Psychophysics Toolbox version 3 (Brainard, 1997; Pelli, 1997), and were presented on an LCD screen $(70 \times 39.5 \mathrm{~cm}, 1920 \times 1080$ pixel resolution, $60 \mathrm{~Hz}$ refresh rate) which the participants viewed through a mirror mounted above their eyes at an effective distance of $156 \mathrm{~cm}$. Participants' reports were recorded using a fiber-optical MR-compatible response box (Cedrus Lumina LSC-400B).

To confirm whether participants maintained central fixation during the acquisition session, individually calibrated eye tracking data (sample frequency of $500 \mathrm{~Hz}$ ) were recorded inside the scanner using Eyelink 1000 software (SR Research, Ottawa, Ontario, Canada).

The stimuli and the procedure used for each scanning run are described below.

\subsection{1. $h M T+$ Functional Localizer}

The goal of this run was to find the subject-specific location of our region-of-interest (ROI), the hMT+ complex. For this purpose, 350 white dots (diameter of $0.08^{\circ}$ ) were shown inside a $9^{\circ}$ circular aperture at the center of a black screen (Fig. 2A). Blocks of $15 \mathrm{~s}$ with moving dots $\left(3^{\circ} / \mathrm{s}\right)$, in all eight cardinal and intermediate directions, were interleaved with blocks of $15 \mathrm{~s}$ with static dots. A central red cross (width of $0.67^{\circ}$ ) was displayed as a fixation target at the visual midline. The run lasted for 3.2 min, composed by six motion condition repetitions interleaved with the static dot condition.

\subsubsection{Testing runs}

We used moving plaids (superposition of two moving gratings Fig. 2B) which are inherently bistable stimuli (if no texture is added):

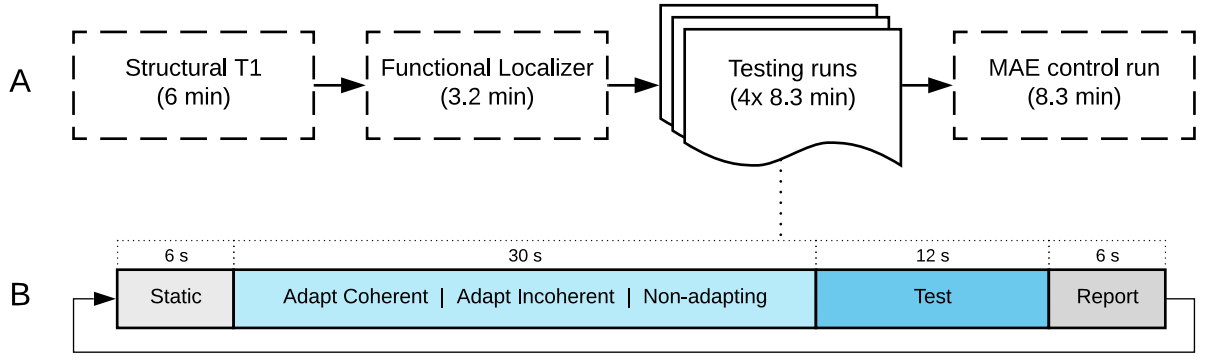

Fig. 1. Experimental design. A) Diagram of the scanning session. B) Protocol scheme of each trial of the testing runs. 

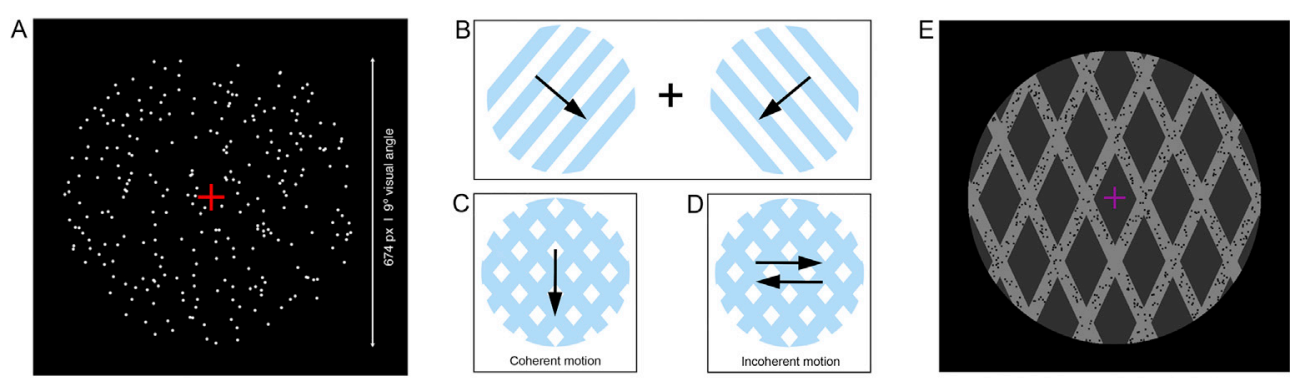

Fig. 2. Stimuli used in the experiment. A) Functional localizer used to map the hMT+ ROI in each participant's visual cortex. Moving dots were shown inside a circular aperture at the center of a black screen. Panels B, C and D illustrate a plaid stimulus. By superimposing two gratings (B), moving orthogonally to the lines, a bistable stimulus is created, which can be perceived moving coherently (C) as a single surface or incoherently (D) as two separate surfaces sliding over each other. Arrows illustrate the direction of perceived motion. E) Plaid stimulus used during the testing runs. Depending on the moving dots' direction the otherwise ambiguous stimulus was readily perceived as a plaid moving coherently or incoherently.

they can be perceived moving coherently (Fig. 2C) or incoherently (Fig. 2D), and as such, the stimulus is intrinsically ambiguous (Adelson and Movshon, 1982). The testing runs were used to measure the blood-oxygen-level dependent (BOLD) fMRI signal in hMT+ during adaptation to the coherent and incoherent motion percepts and during the potential subsequent perception of MAE.

To force the perception of one of the two possible motion interpretations, we added dots to the plaid to disambiguate motion (Fig. 2E). Depending on the relative percentage of dots that move vertically or horizontally, we were able to induce a given perceptual interpretation to the participant. During adaptation to coherent motion all the dots moved downward, forcing the coherent percept, and during adaptation to incoherent motion the dots moved left or right in each grating, forcing the incoherent percept of the plaid. In Table 1, we summarize the stimulus properties, which have been optimized to guarantee an unambiguous perceptual interpretation of each type of motion (coherent and incoherent).

A non-adapting condition was used as control for the adaptation effects. In this condition, the motion percept is made to change at each volume scanning time (TR, $1.5 \mathrm{~s}$ ). The dots direction of motion changes at every volume creating alternated coherent or incoherent motion in one of the four cardinal directions. An attention task was used to ensure that participants were looking attentively at the fixation cross and also paying attention to a motion-unrelated feature. This attention task was used to control for MAE contamination due to shifts in arousal or by selective attention, as previously described (Castelo-Branco et al., 2009; Hogendoorn and Verstraten, 2013). From one to four times in each trial, the central fixation cross became slightly larger for just $250 \mathrm{~ms}\left(0.67^{\circ}\right.$ to $0.80^{\circ}$ ). The task was to detect and count these changes during the trial, and to report this number at the end of the trial. Importantly, global motion perception (including MAE) is in general spatially uniform and equally distributed within perceptually segmented surfaces, specially when stimuli are viewed centrally (Castelo-Branco et al., 2009; Kozak and Castelo-Branco, 2009).

Table 1

Summary of the plaid stimulus properties.

\begin{tabular}{ll}
\hline Angle of gratings relative to horizontal $\left(^{\circ}\right)$ & 65 \\
Duty cycle (\%) & 25 \\
Aperture diameter $\left({ }^{\circ}\right.$ visual angle) & 9 \\
Screen background color (rgb) & $(0,0,0)$ \\
Plaid background color (rgb) & $(50,50,50)$ \\
Gratings color (rgb) & $(130,130,130)$ \\
Spatial frequency (cycle ${ }^{\circ}$ visual angle) & 0.625 \\
Motion speed $\left({ }^{\circ}\right.$ visual angle/s) & 1.6 \\
Number of dots & 800 \\
Dots color (rgb) & $(20,20,20)$ \\
Dots size $\left({ }^{\circ}\right.$ visual angle) & 0.05 \\
Dots horizontal speed $\left({ }^{\circ}\right.$ visual angle/s) & 2.4 \\
Dots vertical speed $\left({ }^{\circ}\right.$ visual angle/s) & 4 \\
Fixation cross width $\left({ }^{\circ}\right.$ visual angle) & 0.67 \\
\hline
\end{tabular}

Each trial (described schematically in Fig. 1B) included a static condition (static figure of the plaid stimulus) of $6 \mathrm{~s}$, followed by an adapting or non-adapting moving condition of $30 \mathrm{~s}$, a test condition of $12 \mathrm{~s}$, and a report period of $6 \mathrm{~s}$. During the report period the participant used one of the four buttons in the response box to report the number of cross size changes. Within the report block, feedback (correct/incorrect) was provided by briefly changing the color of the fixation cross to green or red, respectively.

In each of the four runs, we included three trials of each type: adaptation to coherent, adaptation to incoherent and non-adapting. The sequence of trials was randomized between runs and participants.

\subsubsection{MAE control run}

The goal of this run was to obtain a behavioral metric about the perception of MAE. The protocol was alike the testing runs, with one additional task for the participant. After the attention task report, the participant was instructed to report if illusory motion was perceived during the "Test" period. The fMRI data of this run is not used since the participant is paying attention to a motion-related feature during the "Test" period. This run was performed within the scanner to maintain identical stimulation conditions during both imaging and behavioral data acquisition.

\section{3. fMRI data acquisition}

Scanning was performed on a 3T Siemens Magnetom Tim Trio scanner, at the Institute of Nuclear Sciences Applied to Health (ICNAS), Portugal. Using a 12-channel head coil, the scanning session started with the acquisition of one 3D anatomical magnetization-prepared rapid acquisition gradient echo (MPRAGE) pulse sequence (TR $=2530 \mathrm{~ms}$, echo time $(\mathrm{TE})=3.42 \mathrm{~ms}$, flip angle $=7^{\circ}, 176$ slices, voxel size $1.0 \times 1.0 \times 1.0 \mathrm{~mm}$, field of view $($ FOV $)=256 \times 256 \mathrm{~mm})$. Afterwards, six functional runs were acquired using a $\mathrm{T} 2{ }^{*}$-weighted gradient echo planar imaging (EPI) sequence. These consisted of 128 volumes in the first run and 332 volumes in the following (TR $=1500 \mathrm{~ms}, \mathrm{TE}=30 \mathrm{~ms}$, flip angle $=75^{\circ}, 27$ interleaved slices without gap, voxel size $3.5 \times 3.5 \times 3.5 \mathrm{~mm}, \mathrm{FOV}=235 \times 235 \mathrm{~mm}$ ), yielding total coverage of the occipital and parietal lobes and partial coverage of frontal and temporal lobes. In total, the duration of the scanning session was of $50 \mathrm{~min}$.

\section{4. fMRI data processing}

Data processing was performed on BrainVoyager QX 2.8 (Brain Innovation, The Netherlands), automated using custom MATLAB scripts. Pre-processing included slice-scan time correction, 3D head-motion correction and temporal high-pass filtering (GLM-Fourier, 2 cycles). Data were normalized to Talairach space (Talairach and Tournoux, 1988). Activation maps were created using a General Linear Model (GLM), with predictors for each experimental condition and confound 
predictors from six detrended head motion parameters ( 3 translation, 3 rotation) and spikes (Satterthwaite et al., 2013).

\subsection{Statistical data analysis}

\subsection{1. $h M T+$ LOCALIZATION}

The left and right hMT + were functionally localized in every participant with an individual GLM analysis of the localizer run. The region was defined as the voxels in the posterior middle temporal region responding significantly to the contrast "Moving $>$ Static". The individual statistical maps were limited at threshold value of $p=0.05$, Bonferroni-corrected. For the following analyses, the left and right ROIs were used combined as a single region, which we designate by bilateral hMT + .

\subsubsection{Testing runs data analysis}

The time course of the bilateral hMT+ in each testing run was extracted to calculate the percent BOLD signal variation, using as baseline the average of the values during the "Static" condition. These normalized time courses were split into trial type and averaged across trials and participants, resulting in a group analysis of the BOLD activation in our ROI.

A fixed effects (FFX) GLM analysis was first computed for each participant's testing runs. From this analysis, we extracted the beta values of the bilateral hMT + for three contrasts: "Test after Coherent vs. Adapt Coherent", "Test after Incoherent vs. Adapt Incoherent", "Test after Nonadapting vs. Non-adapting". Gathering the beta values of the three contrasts of each participant, a repeated measures one-way analysis of variance (ANOVA) with Greenhouse-Geisser correction was performed, as well as a Bonferroni's corrected multiple comparisons test, with individual variances computed for each comparison. The aim of this analysis was to verify how much the BOLD signal decreased from the motion conditions to the test conditions and whether this decrease depended or not on the type of motion. Importantly, we avoided simple slope comparisons: we compared the magnitude of signal decrease in each "Test" condition relative to each motion condition's own response amplitude, which is an intrinsic control for condition-specific adaptation-related signal decreases.

To further analyze the BOLD signal dynamics during the "Test" condition and its modulation dependence on the preceding adapting condition, we have calculated the Area Under Curve (AUC) of the signal variation during the "Test" condition for the pooled data of the testing runs per participant. Specifically, we considered eight data points from the "Test" condition, plus one point before and after the condition, totaling 10 data points for calculating the AUC using the trapezoid rule. Before this calculation, we have compensated for the different initial level of activation (at the beginning of the "Test" period) for each of the three trial types, by subtracting the average value of the three data points before the "Test" condition of each trial type. In this way, we assured that the AUC differences in the "Test" condition did not depend on the initial signal value, but rather on the stronger or weaker decrease of activity in hMT + . Gathering the AUC data of each trial type and participant, a repeated measures one-way ANOVA with Greenhouse-Geisser correction was performed, as well as a Bonferroni's corrected multiple comparisons test, with individual variances computed for each comparison.

\section{Results}

\section{1. $h M T+$ Localization}

The GLM statistical maps of the localizer run revealed significant activation in the bilateral hMT+ complex of each participant. Using the contrast between moving and static dots, we were able to define left and right hMT + for all participants. The average Talairach coordinates ( $\mathrm{x}, \mathrm{y}$, z) for the left hMT + are $(-44 \pm 4,-68 \pm 4,1 \pm 5)$ and for the right hMT + are ( $43 \pm 4,-69 \pm 4,-1 \pm 4)$. The probability map of location of hMT+ is shown in Fig. 3.

\subsection{Visual motion adaptation}

After defining the bilateral hMT + ROIs at the single-subject level, the time courses of each participant's testing runs were extracted. The BOLD signal variation was calculated using the average value during the static condition as baseline. Fig. 4 shows the average time course of the response in bilateral hMT+, for all participants and runs, for the three trial types: adaptation to coherent motion, adaptation to incoherent motion and non-adapting. The hMT+ response to the non-adapting condition was the strongest, followed by the response to the incoherent percept and then to the coherent percept. When the plaid stopped moving (the test condition started), hMT + activity decreased on the three trial types, as expected. Note, however, that this decrease was stronger when preceded by non-adapting motion than by both types of adapting motion. Importantly, the signal decreased less after the perception of coherent than after incoherent motion.

The differences in the decrease of the BOLD signal during the test period were also evident at the level of GLM statistical analysis. The beta values of the contrast between the test condition and each motion condition were estimated per participant and compared. Accordingly, the more negative the contrast beta value, the stronger the hMT+ signal variation. As illustrated in Fig. 5, there was a statistically significant difference between the contrasts as determined by one-way repeated measures ANOVA $\left(F(1.52,28.83)=44.93, p=1.18 \times 10^{-8}\right)$. The contrast beta values were significantly higher for the coherent trials $(-0.84 \pm 0.08, p$ (corrected $\left.)=1.20 \times 10^{-6}\right)$ and the incoherent trials $\left(-1.01 \pm 0.07, p(\right.$ corrected $\left.)=6.08 \times 10^{-6}\right)$ when compared with the non-adapting trials $(-1.26 \pm 0.08)$. Furthermore, there was a statistically significant difference between the beta values of the coherent and incoherent trials $\left(p(\right.$ corrected $\left.)=8.40 \times 10^{-4}\right)$.

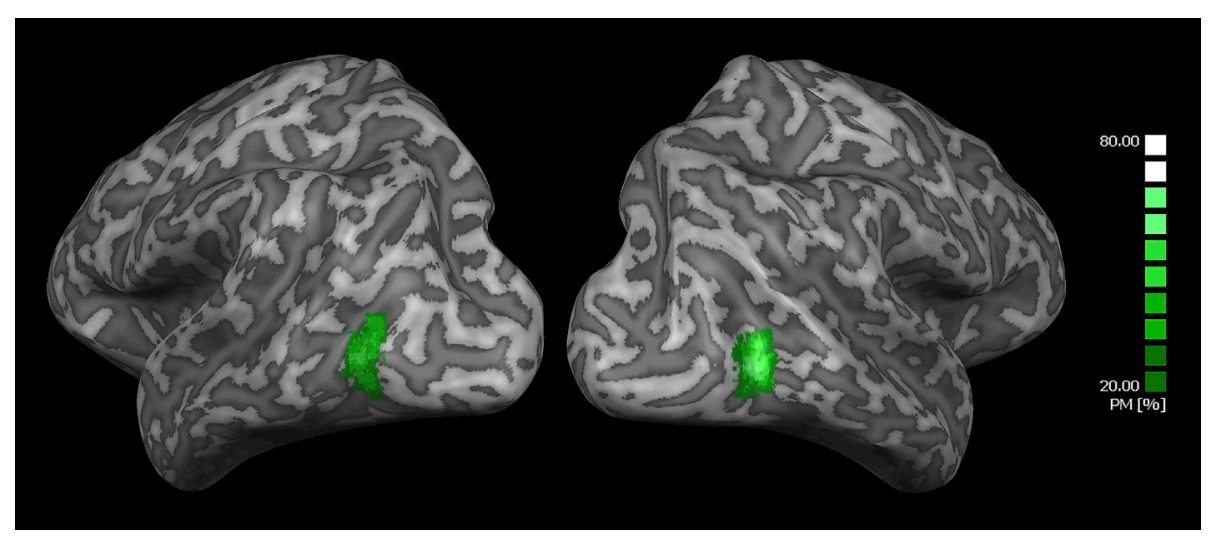

Fig. 3. Probability map of the bilateral hMT + ROIs, which were defined at the single-subject level for all participants. 


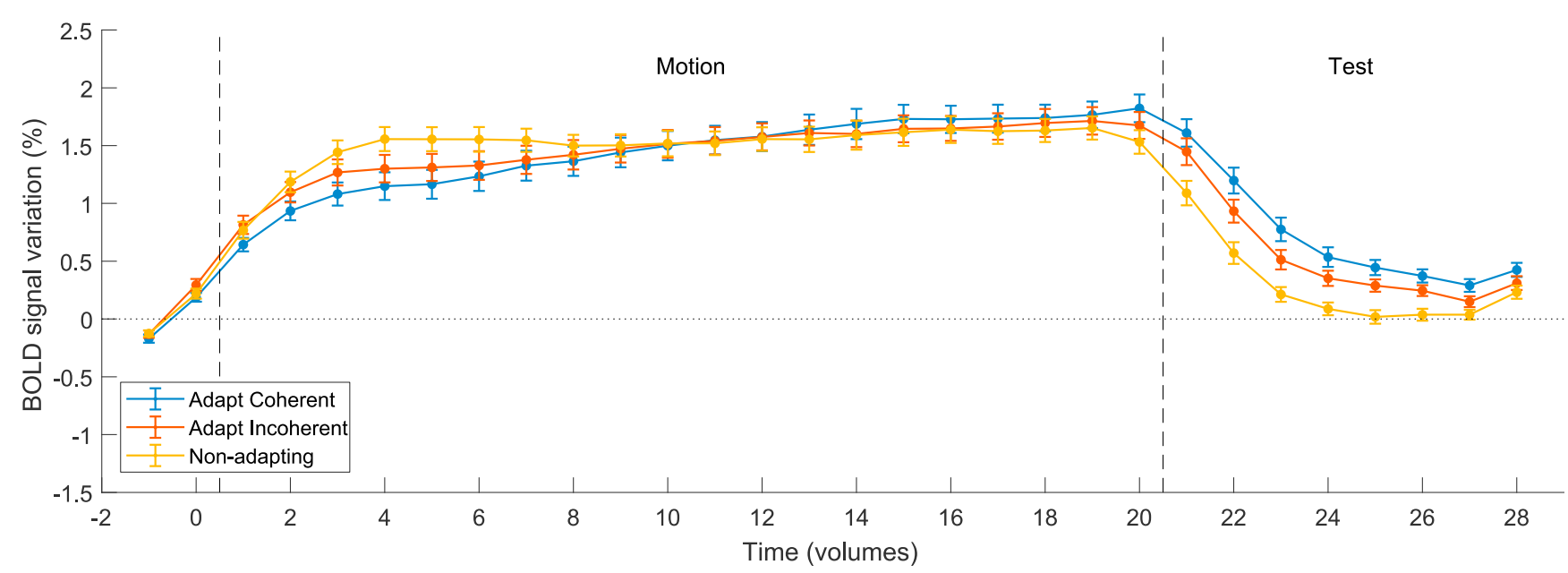

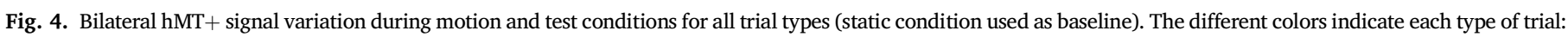

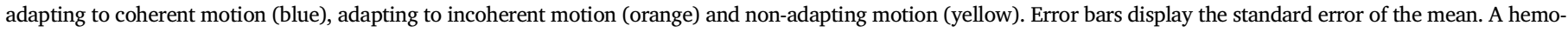
dynamic delay of 3 volumes was considered.

We have analyzed with further detail the hMT + time course during the test condition. In Fig. 6, we present three line plots, each comparing the time course of the response of two of the three trial types. We used the AUC as a metric of the hMT + activity during the test period after each motion condition (scaled to compensate for different initial value of the curves). The differences between the areas were statistically significant (Fig. 6D), as revealed by one-way repeated measures ANOVA $(F(2,38)=30.84$, $p=1.10 \times 10^{-8}$ ). The hMT + activity was significantly higher during the test period after adaptation to coherent motion $(6.19 \pm 0.39$, $p$ (corrected $)=2.12 \times 10^{-6}$ ) and to incoherent motion $(5.41 \pm 0.32$, $p$ (corrected) $=1.46 \times 10^{-4}$ ) compared to the activity during the test period after non-adapting motion $(4.14 \pm 0.29)$. Furthermore, the difference in activity during the test period after the adaptation to coherent and incoherent motion was significant $\left(p\right.$ (corrected) $=2.35 \times 10^{-2}$ ).

Regarding the attention task, although participants reported that it was relatively demanding, they still answered correctly, on average, 6 out of 9 times per run $(S D=2)$.

\subsection{Mae control run}

We analyzed the participants' reports regarding the perception of illusory motion during the test period that followed each plaid motion percept. The results are shown in Fig. 7. After the coherent motion percept, the number of MAE reports was considerably higher than after the incoherent motion percept ( 40 vs. 19 out of 57 trials). After the nonadapting motion, none of the participants reported any percept of illusory motion. These results come from 19 participants, due to missing button press data from one participant.

\subsection{Fixation data}

Participants' fixation was monitored by visual inspection during all the scanning sessions and by the eye tracking system. Afterwards, we have analyzed the eye tracking data of 10 participants as a quality control of the experiment, using a MATLAB custom script. It was not possible to record eye tracking data of the remaining 10 participants due to eye tracker system incompatibilities. We defined a rectangular area of interest $\left(4^{\circ} \times 4^{\circ}\right)$ in the center of the image. The average time of successful fixation (inside this area of interest) was $81 \%(S D=11 \%)$.

\section{Discussion}

In this study, we tested whether visual adaptation occurs for both coherent and incoherent plaid motion perception and investigated its respective strength at the neurophysiological level. We found that hMT + activity is differentially modulated by adaptation depending on the perceived type of motion. The strength of the visual adaptation effect depended on whether subjects integrated all motion signals into a single surface or whether they segregated signals and perceived two transparent surfaces, while being significant in both cases. This result suggests that the identified asymmetric adaptation to each perceptual interpretation can help explain perceptual dynamics of bistability and contribute to its modeling.

The discovery that visual adaptation due to incoherent motion perception exists shows that it also plays a role in controlling perceptual duration, albeit in an asymmetric manner. We could directly compare its strength with the strength of adaptation due to coherent motion. The latter is stronger, thereby leading to larger weights in models of

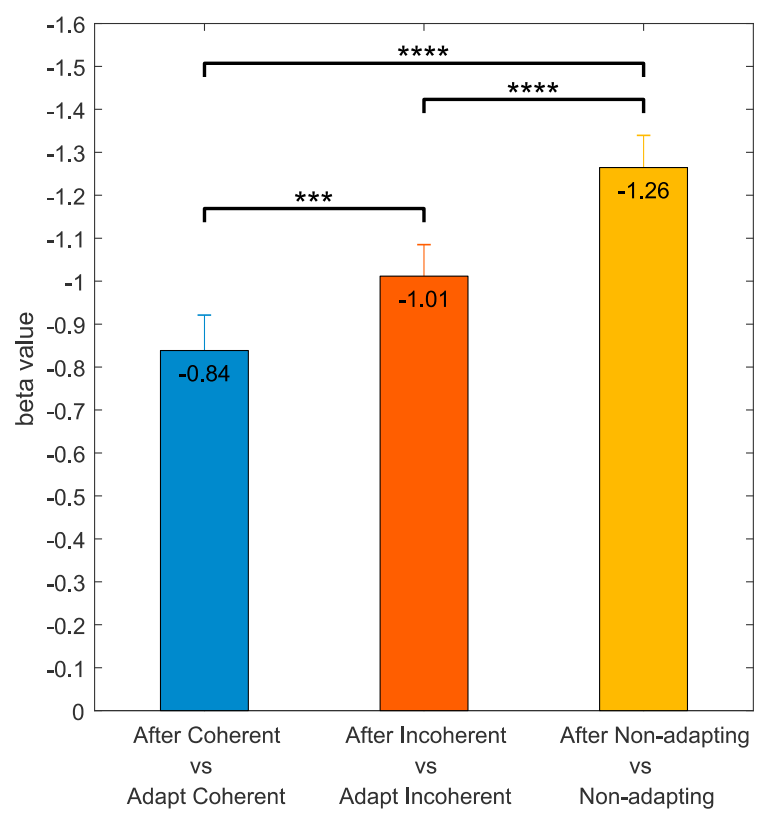

Fig. 5. Beta values for each trial type, by contrasting the hMT + activity during the test condition with the preceding motion condition. The bars represent this contrast for the coherent (in blue), incoherent (in orange) and non-adapting (in yellow) trials, across all participants. Error bars show the standard error of the mean. A group statistical analysis was performed on the beta values of each contrast, revealing significant group differences between all (***p $\leq 0.001$, $* * * * \mathrm{p} \leq 0.0001$, Bonferroni-corrected). 
A

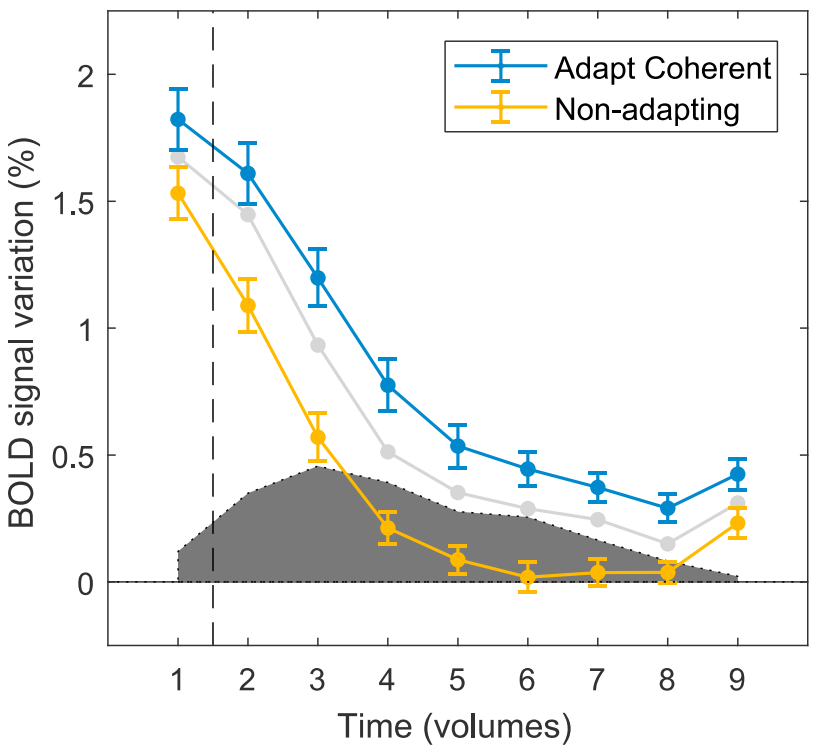

C

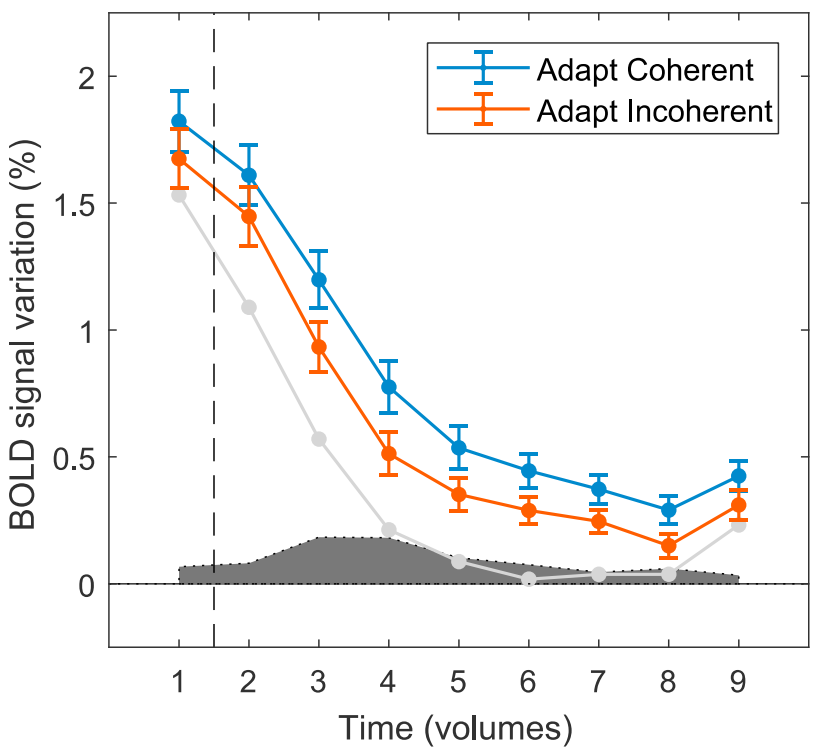

B

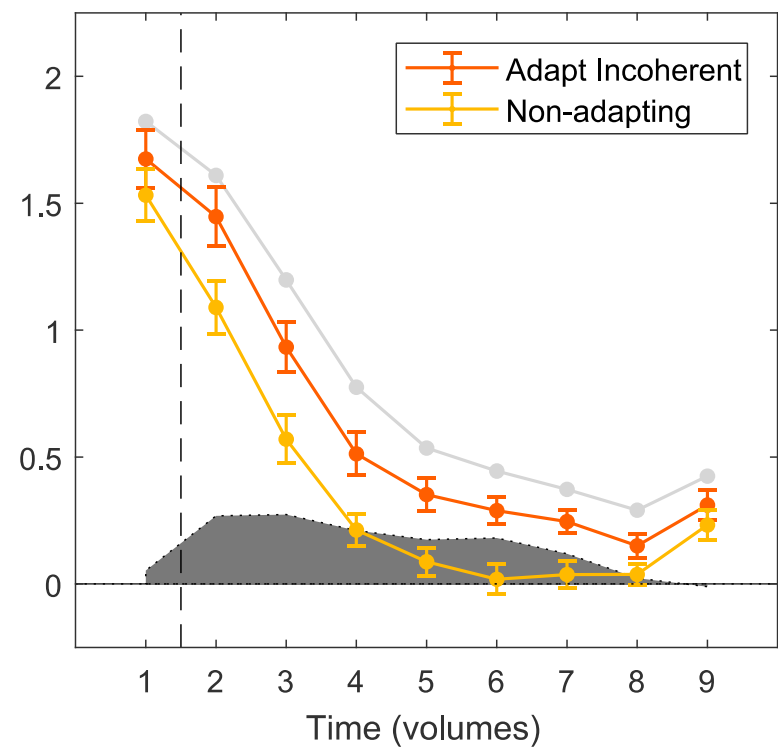

D

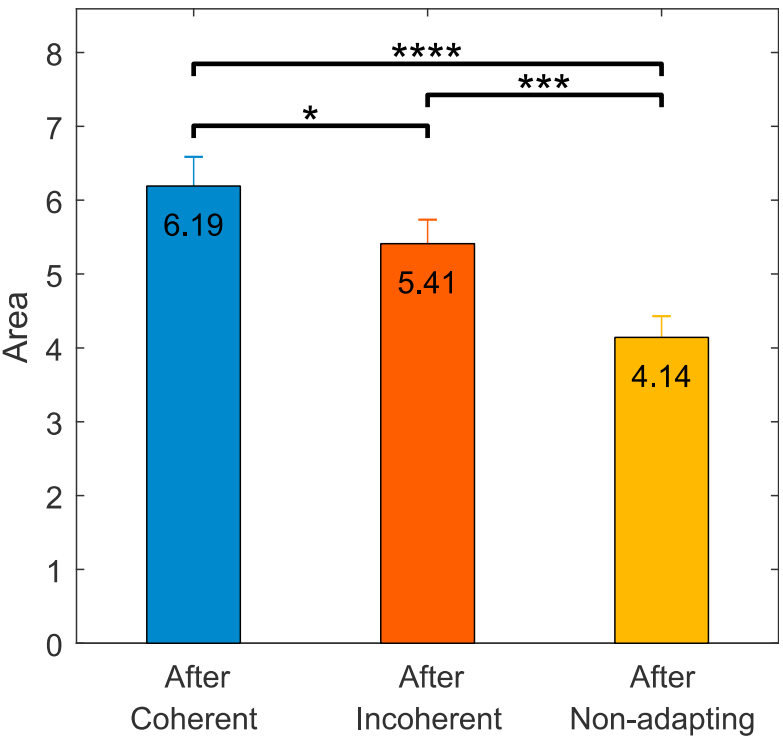

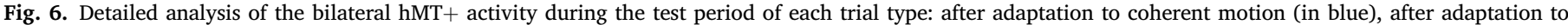

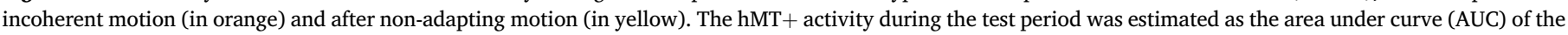

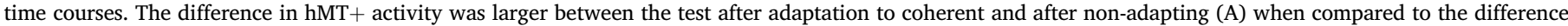

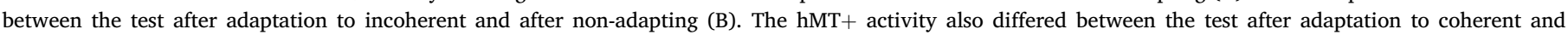

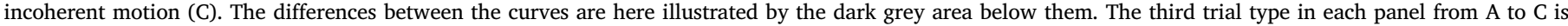

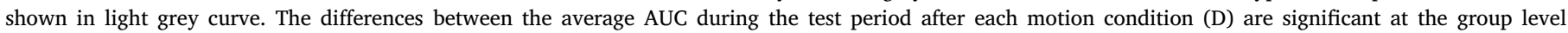

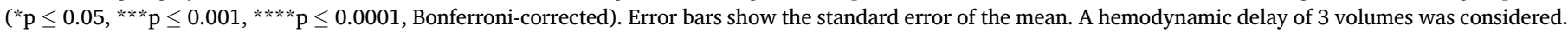

perceptual bistability. As suggested before by Huguet et al. (2014), perceptual bistability models should be adjusted regarding each percept dominance duration and probability. For instance, if a stimulus leads to distinct mean dominance durations and probabilities of each percept, this suggests asymmetric inhibition and adaptation-driven input strength. Our evidence for asymmetric adaptation reinforces the idea that asymmetric weights do control perceptual choice and duration, which can help refine computational models based on modulation of adaptation.

The localizer implemented in this experiment allowed us to unequivocally define our region of interest, for each participant. We verified a substantial variability in the hMT+ location across participants, both from the probability maps of Fig. 3 and the standard deviation of the Talairach coordinates. These results prompted that subsequent analysis should be based on the BOLD signal extracted from subject-specific ROIs, rather than from average group ROIs. Even so, the average coordinates match previous studies that localize the same region (Duarte et al., 2017; Sousa et al., 2016; Tootell et al., 1995b).

The fact that non-adapting motion perception is accompanied by the highest hMT+ activation level is likely to be due to more motion variation of the stimulus, resulting in the activation of multiple direction-selective cell assemblies (Fig. 4). The different level of activation as a response to the coherent and incoherent motion percept (higher for the incoherent) is 


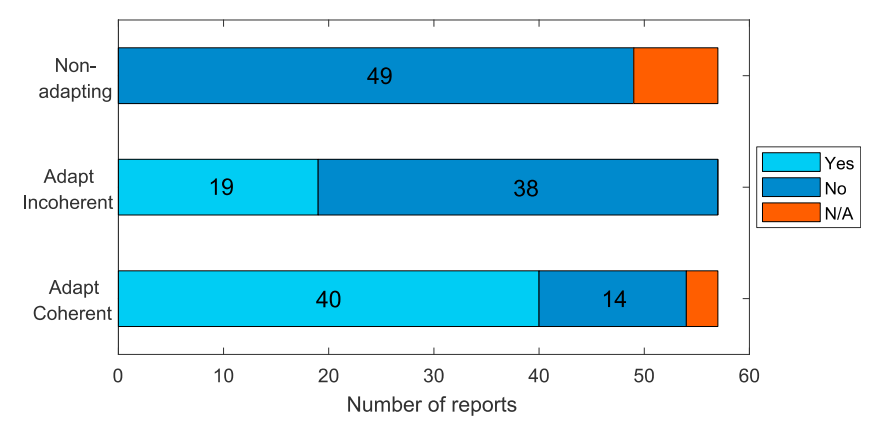

Fig. 7. Participants' reports of the MAE control run regarding the perception of motion after-effect during the test condition.

also consistent with this explanation, as already shown in previous research (Castelo-Branco et al., 2002; Duarte et al., 2017). Note also the curve crossing midway through the motion stimulation period, consistent with long visual stimulation, as in (Hogendoorn and Verstraten, 2013), and with oscillatory models of slow adaptation (Huguet et al., 2014).

When the stimulus stops moving, the decrease of hMT + activity depends on the preceding motion condition. We observed higher $\mathrm{hMT}+$ activity during the test (static) period after visual adaptation to both coherent and incoherent motion when compared to non-adapting motion. This hMT+ response, potentially related with the MAE phenomena (Anstis et al., 1998; Mather et al., 2008), was stronger after adaptation to coherent than to incoherent motion. Nevertheless, a clear adaptation response could be retrieved for the latter condition. Accordingly, adaptation to coherent motion causes the perception of MAE in most of the trials, while adaptation to incoherent motion does not (Fig. 7). The presence of an attention demanding task helped to control for shifts in arousal and/or selective attention (Castelo-Branco et al., 2009).

The analyses on the beta values of the GLM (Fig. 5) and on the AUC of activity during the test period (Fig. 6) reveal matching and significant differences between the three trial types. Our findings further demonstrate that the adaptation effect in hMT+ is indeed different for the two percepts. Our results are in agreement with previous studies which suggest an increase in hMT+ activity due to the MAE (Castelo-Branco et al., 2009; Tootell et al., 1995a). Furthermore, the different levels of MAE reflected on hMT+ activity suggest the presence of tuned disinhibition for different neuronal populations, according to the perceived type of plaid motion (Kohn and Movshon, 2003; Petersen et al., 1985).

We hypothesize that coherent motion engages activation and adaptation of a neuronal population which is selective for a single direction of motion, but also the inhibition of the population selective for the opposite direction. On the other hand, incoherent motion engages activation and adaptation of two neuronal populations which are selective for each surface's direction of motion. Since these are tuned for opposing directions, we hypothesize a cross-inhibitory mechanism that leads the two populations to be activated and inhibited at the same time during the perception of incoherent motion. When the stimulus stops moving, these populations disinhibit, and BOLD signal persistence is evident. Our results, which report higher signal persistence for the coherent than for the incoherent motion percept, support the hypothesis of this crossinhibitory mechanism. While after coherent motion we expect to measure the response of a neuronal population that was strongly inhibited (but not activated during coherent motion), after incoherent motion we expect to measure a weaker response from the two cross-inhibited neuronal populations (which were also activated during incoherent motion), leading to a lower BOLD signal persistence. Despite showing different BOLD signal persistence during the test period, both conditions still show activity above the control condition, i.e. non-adapting motion, strongly suggesting that there is indeed an inhibitory mechanism responsible for the activity during the test period.

As a fundamental novelty of this study, our data demonstrates that the $\mathrm{hMT}+$ region adapts not only to the perception of coherent motion, as previously shown (Huk and Heeger, 2002), but also adapts to the perception of incoherent motion. Importantly, the stimulus used for the coherent and incoherent motion is essentially the same: the difference is the perceived type of motion. We show for the first time that hMT + adapts to the perceived coherent motion and to the perceived incoherent motion, but at a distinct level. During the visualization of bistable plaid stimuli, this would imply that hMT + is constantly adapting and that the level of adaptation would vary according to the perceived type of motion.

In a recent study, Patterson et al. (2014) suggested that the ability of $\mathrm{hMT}+$ neurons to integrate motion signals is impeded by adaptation at earlier visual processing levels. Our study does not focus on adaptation in low-level visual areas, such as V1, but rather on adaptation to moving plaids which alters the response in hMT + . Interestingly, both results could be integrated in the framework of a two-stage model such as the one proposed by Patterson et al. (2014). In this case, the first stage would model adaptation to local motion and the second stage would model adaptation to global motion. The computation of these stages might be achieved separately in lower and higher level visual areas or both within hMT + itself.

In sum, we suggest that our results are relevant to the understanding of perceptual decision, mainly since visual adaptation has been pointed out as one of the possible causes of perceptual bistability (Huguet et al., 2014; Kohn and Movshon, 2004; Long and Toppino, 2004). Our results contribute to existing models of perceptual bistability, since these should now consider distinct adaptation weights depending on the type of percept (incoherent vs. coherently moving surfaces).

\section{Conclusions}

Activity in hMT+ reflects different levels of measurable visual adaptation to both coherent and incoherent percepts of bistable plaid motion. These results demonstrate asymmetric adaptation, which may contribute to understand how the visual system controls perceptual decision based on low-level mechanisms. Future studies should investigate how the duration of each percept during bistability is controlled by such asymmetric adaptation weights.

\section{Funding}

This research work was funded by the Portuguese Foundation for Science and Technology (FCT) (grants: COMPETE UID/NEU/04539/2013, COMPETE POCI-01-0145-FEDER-007440) and by the BIAL project 207/16.

\section{Acknowledgements}

We would like to thank the participants for their involvement in this study. We are also very grateful to Carlos Ferreira and Sónia Afonso for the help with fMRI setup and scanning.

\section{References}

Adelson, E.H., Movshon, J.A., 1982. Phenomenal coherence of moving visual patterns. Nature 300, 523-525. https://doi.org/10.1038/300523a0.

Albright, T.D., Stoner, G.R., 1995. Visual motion perception. Proc. Natl. Acad. Sci. U. S. A 92, 2433-2440. https://doi.org/10.1073/pnas.92.7.2433.

Anstis, S., Verstraten, F.A., Mather, G., 1998. The motion aftereffect. Trends Cognit. Sci. 2, 111-117. https://doi.org/10.1016/S1364-6613(98)01142-5.

Brainard, D.H., 1997. The psychophysics Toolbox. Spatial Vis. 10, 433-436. https://doi.org/ 10.1163/156856897X00357.

Castelo-Branco, M., Formisano, E., Backes, W., Zanella, F., Neuenschwander, S., Singer, W., Goebel, R., 2002. Activity patterns in human motion-sensitive areas depend on the interpretation of global motion. Proc. Natl. Acad. Sci. Unit. States Am. 99, 13914-13919. https://doi.org/10.1073/pnas.202049999.

Castelo-Branco, M., Kozak, L.R., Formisano, E., Teixeira, J., Xavier, J., Goebel, R., 2009. Type of featural attention differentially modulates hMT + responses to illusory motion

aftereffects. J. Neurophysiol. 102, 3016-3025. https://doi.org/10.1152/jn.90812.2008.

Culham, J.C., Dukelow, S.P., Vilis, T., Hassard, F. a., Gati, J.S., Menon, R.S., Goodale, M. a., 1999. Recovery of fMRI activation in motion area MT following storage of the motion aftereffect. J. Neurophysiol. 81, 388-393. https://doi.org/10.1152/jn.1999. 81.1.388. 
Duarte, J.V., Costa, G.N., Martins, R., Castelo-Branco, M., 2017. Pivotal role of hMT + in long-range disambiguation of interhemispheric bistable surface motion. Hum. Brain Mapp. 38, 4882-4897. https://doi.org/10.1002/hbm.23701.

Gibson, J.J., 1937. Adaptation, after-effect, and contrast in the perception of tilted lines. II. Simultaneous contrast and the areal restriction of the after-effect. J. Exp. Psychol. 20, 553-569. https://doi.org/10.1037/h0057585.

Grill-Spector, K., Henson, R., Martin, A., 2006. Repetition and the brain: neural models of stimulus-specific effects. Trends Cognit. Sci. 10, 14-23. https://doi.org/10.1016/j.tics. 2005.11.006.

Grill-Spector, K., Malach, R., 2001. fMR-adaptation: a tool for studying the functional properties of human cortical neurons. Acta Psychol. 107, 293-321. https://doi.org/ 10.1016/S0001-6918(01)00019-1.

Hershenson, M., 1989. Duration, time constant, and decay of the linear motion aftereffect as a function of inspection duration. Percept. Psychophys. 45, 251-257. https://doi.org/ 10.3758/BF03210704.

Hogendoorn, H., Verstraten, F.A.J., 2013. Decoding the motion aftereffect in human visual cortex. Neuroimage 82, 426-432. https://doi.org/10.1016/ j.neuroimage.2013.06.034.

Huguet, G., Rinzel, J., Hupé, J.-M., 2014. Noise and adaptation in multistable perception: noise drives when to switch, adaptation determines percept choice. J. Vis. 14, 1-24. https://doi.org/10.1167/14.3.19.

Huk, A., Heeger, D., 2002. Pattern-motion responses in human visual cortex. Nat. Neurosci. 5, 72-75. https://doi.org/10.1038/nn774.

Kar, K., Krekelberg, B., 2016. Testing the assumptions underlying fMRI adaptation using intracortical recordings in area MT. Cortex 80, 21-34. https://doi.org/10.1016/j. cortex.2015.12.011.

Kohn, A., 2007. Visual adaptation: physiology, mechanisms, and functional benefits. J. Neurophysiol. 97, 3155-3164. https://doi.org/10.1152/jn.00086.2007.

Kohn, A., Movshon, J.A., 2004. Adaptation changes the direction tuning of macaque MT neurons. Nat. Neurosci. 7, 764-772. https://doi.org/10.1038/nn1267.

Kohn, A., Movshon, J.A., 2003. Neuronal adaptation to visual motion in area MT of the macaque. Neuron 39, 681-691. https://doi.org/10.1016/S0896-6273(03)00438-0.

Kolster, H., Peeters, R., Orban, G.A., 2010. The retinotopic organization of the human middle temporal area MT/V5 and its cortical neighbors. J. Neurosci. 30, 9801-9820. https://doi.org/10.1523/jneurosci.2069-10.2010.

Kozak, L.R., Castelo-Branco, M., 2009. Peripheral influences on motion integration in foveal vision are modulated by central local ambiguity and center-surround congruence. Investig. Ophthalmol. Vis. Sci. 50, 980-988. https://doi.org/10.1167/ iovs.08-2094.

Krekelberg, B., Boynton, G.M., van Wezel, R.J.A., 2006. Adaptation: from single cells to BOLD signals. Trends Neurosci. 29, 250-256. https://doi.org/10.1016/ j.tins.2006.02.008.

Larsson, J., Harrison, S.J., 2015. Spatial specificity and inheritance of adaptation in human visual cortex. J. Neurophysiol. 114, 1211-1226. https://doi.org/10.1152/jn. 00167.2015.

Leopold, D.A., Logothetis, N.K., 1999. Multistable phenomena: changing views in perception. Trends Cognit. Sci. https://doi.org/10.1016/S1364-6613(99)01332-7.

Long, G.M., Toppino, T.C., 2004. Enduring interest in perceptual ambiguity: alternating views of reversible figures. Psychol. Bull. 130, 748-768. https://doi.org/10.1037/ 0033-2909.130.5.748.
Mather, G., Pavan, A., Campana, G., Casco, C., 2008. The motion aftereffect reloaded. Trends Cognit. Sci. 12, 481-487. https://doi.org/10.1016/j.tics.2008.09.002.

Movshon, J.A., Adelson, E.H., Gizzi, M.S., Newsome, W.T., 1985. The analysis of moving visual patterns. In: Chagas, C., Gattass, R., Gross, C. (Eds.), Pattern Recognition Mechanisms. Vatican Press, Rome, pp. 117-151.

Müller, J.R., Metha, A.B., Krauskopf, J., Lennie, P., 1999. Rapid adaptation in visual cortex to the structure of images. Science 285, 1405-1408. https://doi.org/10.1126/ science.285.5432.1405.

Oldfield, R.C., 1971. The assessment and analysis of handedness: the Edinburgh inventory. Neuropsychologia 9, 97-113. https://doi.org/10.1016/0028-3932(71) 90067-4.

Patterson, C.A., Wissig, S.C., Kohn, A., 2014. Adaptation disrupts motion integration in the primate dorsal stream. Neuron 81, 674-686. https://doi.org/10.1016/j.neuron. 2013.11.022

Pelli, D.G., 1997. The VideoToolbox software for visual psychophysics: transforming numbers into movies. Spatial Vis. 10, 437-442. https://doi.org/10.1163/156856897X00366.

Petersen, S.E., Baker, J.F., Allman, J.M., 1985. Direction-specific adaptation in area MT of the owl monkey. Brain Res. 346, 146-150. https://doi.org/10.1016/0006-8993(85) 91105-9.

Satterthwaite, T.D., Elliott, M.A., Gerraty, R.T., Ruparel, K., Loughead, J., Calkins, M.E., Eickhoff, S.B., Hakonarson, H., Gur, R.C., Gur, R.E., Wolf, D.H., 2013. An improved framework for confound regression and filtering for control of motion artifact in the preprocessing of resting-state functional connectivity data. Neuroimage 64, 240-256. https://doi.org/10.1016/j.neuroimage.2012.08.052.

Solomon, S.G., Kohn, A., 2014. Moving sensory adaptation beyond suppressive effects in single neurons. Curr. Biol. 24, R1012-R1022. https://doi.org/10.1016/j.cub.2014. 09.001.

Sousa, T., Direito, B., Lima, J., Ferreira, C., Nunes, U., Castelo-Branco, M., 2016. Control of brain activity in hMT+/V5 at three response levels using fMRI-based neurofeedback/BCI. PLoS One 11. https://doi.org/10.1371/journal.pone.0155961 e0155961.

Talairach, J., Tournoux, P., 1988. Co-planar Stereotaxic Atlas of the Human Brain: 3-D Proportional System: an Approach to Cerebral Imaging, first ed. Thieme Medical Publisher, New York, NY.

Tootell, R.B., Reppas, J.B., Dale, A.M., Look, R.B., Sereno, M.I., Malach, R., Brady, T.J., Rosen, B.R., 1995a. Visual motion aftereffect in human cortical area MT revealed by functional magnetic resonance imaging. Nature. https://doi.org/10.1038/375139a0.

Tootell, R.B., Reppas, J.B., Kwong, K.K., Malach, R., Born, R.T., Brady, T.J., Rosen, B.R., Belliveau, J.W., 1995b. Functional analysis of human MT and related visual cortical areas using magnetic resonance imaging. J. Neurosci. 15, 3215-3230. https://doi.org/10.1523/ jneurosci.15-04-03215.1995.

Tootell, R.B.H., Hadjikhani, N.K., Vanduffel, W., Liu, A.K., Mendola, J.D., Sereno, M.I., Dale, A.M., 1998. Functional analysis of primary visual cortex (V1) in humans. Proc. Natl. Acad. Sci. Unit. States Am. 95, 811-817. https://doi.org/10.1073/pnas.95. 3.811 .

van Wezel, R.J.A., Britten, K.H., 2002. Motion adaptation in area MT. J. Neurophysiol. 88, 3469-3476. https://doi.org/10.1152/jn.00276.2002.

Wang, M., Arteaga, D., He, B.J., 2013. Brain mechanisms for simple perception and bistable perception. Proc. Natl. Acad. Sci. Unit. States Am. 110, E3350-E3359. https://doi.org/10.1073/pnas.1221945110. 